\title{
A Chemical Probe Targeting AAK1 and BMP2K
}

Carrow I. Wells, $\left.{ }^{\dagger \dagger},\right\urcorner$ Rafael M. Couñago, ${ }^{\S, \ngtr}$ Juanita C. Limas, ${ }^{\#}$ Tuanny L. Almeida, ${ }^{\S, \ngtr}$ Jeanette G. Cook, ${ }^{\dagger}$ David H. Drewry, ${ }^{\dagger \dagger}, \neg$ Jonathan M. Elkins, ${ }^{\S, \wedge}$ Opher Gileadi, ${ }^{\S, \wedge}$ Nirav Kapadia, $,{ }^{\dagger \dagger}, \neg$ Álvaro Lorente-Macías, Julie E. Pickett, ${ }^{\dagger \dagger}, \neg$ Alexander Riemen, ${ }^{\dagger}$ Roberta R. Ruela-de-Sousa, ${ }^{\S,}{ }^{\sharp}$ Timothy M. Willson, $\left.{ }^{\dagger \dagger},\right\urcorner$ Cunyu Zhang, "William J. Zuercher, ${ }^{\dagger}, \neg, \quad$ Reena Zutshi, ${ }^{\ddagger}$ Alison D. Axtman $\left.{ }^{*}, \dagger,\right\urcorner$

${ }^{\dagger}$ Structural Genomics Consortium, UNC Eshelman School of Pharmacy, University of North Carolina at Chapel Hill, Chapel Hill, NC, 27599, USA

Division of Chemical Biology and Medicinal Chemistry, UNC Eshelman School of Pharmacy, University of North Carolina at Chapel Hill, Chapel Hill, NC, 27599, USA

${ }^{\S}$ Structural Genomics Consortium, Departamento de Genética e Evolução, Instituto de Biologia, Universidade Estadual de Campinas (UNICAMP), Campinas, SP, 13083-886, Brazil

${ }^{¥}$ Centro de Química Medicinal (CQMED), Centro de Biologia Molecular e Engenharia Genética (CBMEG), UNICAMP, Campinas, SP, 13083-875, Brazil

"Department of Pharmacology, University of North Carolina at Chapel Hill, Chapel Hill, NC, 27599, USA

†Department of Biochemistry and Biophysics, University of North Carolina at Chapel Hill, Chapel Hill, NC, 27599, USA

${ }^{\wedge}$ Structural Genomics Consortium, Nuffield Department of Clinical Medicine, University of Oxford, Old Road Campus Research Building, Oxford, OX3 7DQ, UK

'Departamento de Química Farmacéutica y Orgánica, University of Granada, Granada, 18071, Spain

Lineberger Comprehensive Cancer Center, University of North Carolina at Chapel Hill, Chapel Hill, NC, 27599, USA

\$Luceome Biotechnologies, LLC, Tucson, AZ, 85719, USA

"Platform Technology Sciences, GlaxoSmithKline, Collegeville, PA, 19426, USA

KEYWORDS: AAK1, BMP2K, acylaminoindazole, NAK family, endocytosis, protein kinase

ABSTRACT: Inhibitors based on a 3-acylaminoindazole scaffold were synthesized to yield potent dual AAK1/BMP2K inhibitors. Optimization of this 3-acylaminoindazole scaffold furnished a small molecule chemical probe (SGC-AAK1-1, 25) that is potent and selective for AAK1/BMP2K over other NAK family members, demonstrates narrow activity in a kinome-wide screen, and is functionally active in cells. This inhibitor represents one of the best available small molecule tools to study the functions of AAK1 and BMP2K.

The human protein Ser/Thr kinases Adaptor protein 2-Associated Kinase 1 (AAK1) and BMP-2-Inducible Kinase (BMP2K/BIKE) play critical roles in mediating endocytosis and other key signaling pathways. Both are broadly expressed and are members of the NAK family of human kinases, which also includes Cyclin G-Associated Kinase (GAK) and Myristoylated and Palmitoylated Serine/Threonine Kinase 1 (MPSK1/STK16). The family shares little homology outside of their kinase domains. ${ }^{1}$ AAK 1 and BMP2K are the most closely related, with overall sequence identity of $50 \%$ and kinase domain sequence identity of $74 \% .^{2}$ A key function of AAK1 is regulation of receptor-mediated endocytosis via binding directly to clathrin and phosphorylating the medium subunit of AP2 (adaptor protein 2), which stimulates binding to cargo proteins. ${ }^{3-5}$ AAK1 also modulates the Notch pathway, partially through its phosphorylation of Numb. ${ }^{6,7} \mathrm{BMP} 2 \mathrm{~K}$ plays a role in osteoblast differentiation, is a clathrin-coated vesicle-associated protein, and, like AAK1, also associates with Numb. ${ }^{8,9}$

Due to their many functions, AAK1 and BMP2K have been implicated as potential drug targets for diverse conditions. AAK1 has been linked to diseases affecting the brain such as schizophrenia, Parkinson's disease and amyotrophic lateral sclerosis as well as implicated as a potential anti-viral target for the treatment of Hepatitis C. ${ }^{5,10,11}$ BMP2K has been associated with myopia and evaluated as a potential treatment for HIV. ${ }^{12}$, ${ }^{13}$ A dual AAK1/BMP2K small molecule inhibitor was recently reported as a novel therapeutic to treat neuropathic pain. ${ }^{14}$

X-ray crystal structures for the kinase domains of all NAK family members have been solved and reported. ${ }^{2,15,16}$ Published and novel high-resolution crystal structures of AAK1 and BMP2K reveal target-specific structural features that have enabled our design of specific chemical probes and allowed further 
interrogation of the roles that these kinases play. ${ }^{2}$ Through screening a library of kinase inhibitors (Published Kinase Inhibitor Set, PKIS) via differential scanning fluorimetry (DSF), a chemical starting point showing strong AAK1 binding was identified (Figure 1). ${ }^{17}$ Structure-activity relationships (SAR) were initially established by examining the 3-acylaminoindazole analogs profiled within PKIS. Next, more than 200 analogs were prepared via the route shown in Scheme 1. Briefly, acylation of the indazole aniline with cyclopropanecarbonyl chloride furnished the key intermediate, which was then subjected to Suzuki coupling with a boronic acid. Finally, free anilines on intermediates were reacted with sulfonyl chlorides to yield the desired sulfonamide products. A summary of key analogs prepared to arrive at $\mathbf{1 4}$ is included in Table 1. The compounds were profiled using the AAK1 split luciferase assay developed by Luceome. ${ }^{18,19} \mathbf{1 - 1 4}$ also showed similar affinity for BMP2K (data not shown).
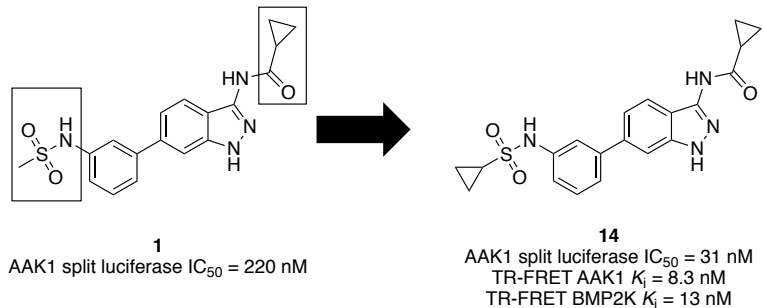

Figure 1. Structures of PKIS hit (1) and key analog 14 with parts of the molecule modified during SAR exploration boxed.

The effects of aryl substitution on the distal aryl ring were explored, including 3- and 4-position substituents of variable size, electronics, and hydrogen-bonding capacity. Sulfonamides, amines, amides, anilines, phenols, acids, and other groups were incorporated in these positions (Table 1). A sulfonamide was found to be optimal in terms of AAK1 inhibition but only when appended at the 3-position of the aryl ring ( 1 versus 2). Sulfonamides with small saturated rings of 5 atoms or fewer and short alkyl chains or alkyl amines were the most potent (1, $\mathbf{1 1}, \mathbf{1 3}, \mathbf{1 4})$. This part of the binding pocket was not tolerant to incorporation of large groups. In addition, 3,5-disubstitution or 3,6-disubstition was disfavored, supporting a small pocket for binding of this aryl ring that is sensitive to even minor changes, such as incorporation of a fluorine in place of a hydrogen (data not shown). Finally, the activity of $\mathbf{1}$ and $\mathbf{8}$ suggests the presence of a hydrogen-bonding network within the ATP-binding pocket that can be accessed by 3-position groups capable of donating a hydrogen. Interestingly, methylation of the aryl 3-nitrogen (11) or insertion of a methylene spacer between the sulfonamide and aryl ring (12) did not decrease potency for AAK1 but rather increased it. These results motivated the design of a range of sulfonamides to further explore the SAR of this part of the molecule.

\section{Scheme 1. Route to prepare compounds in Table $1 .^{\mathrm{a}}$}

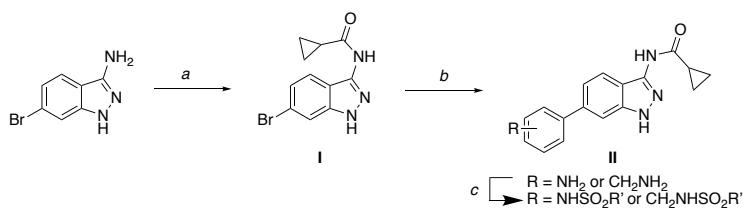

aReagents and conditions: (a) Cyclopropanecarbonyl chloride, pyridine, $0^{\circ} \mathrm{C}\left(68-83 \%\right.$ ) (b) R-boronic acid pinacol ester, $\mathrm{Pd}(\mathrm{dppf}) \mathrm{Cl}_{2}$, $\mathrm{Na}_{2} \mathrm{CO}_{3}$, dioxane, $120^{\circ} \mathrm{C}(82-100 \%)$ (c) R'-sulfonyl chloride, pyridine, $0^{\circ} \mathrm{C}(7-100 \%)$.
The portion of the ATP-binding pocket accessed by the 3position of the indazole was explored through substitution with a variety of groups (Table 2). Compounds in Table 2 were prepared using the route shown in Scheme 1, but the order of the steps was reversed. Thus, a Suzuki reaction was first carried out, and then the indazole aniline was reacted with the corresponding acylating or sulfonylating groups to generate the analogs in Table 2. The indazole 3-position substituent appears to form a polar interaction with the kinase hinge region and is sensitive to structural modifications. The alkyl group was varied from cyclopropyl to methyl, isopropyl, cyclobutyl, phenyl, and cyclopropylmethyl to explore the steric bulk tolerated by the pocket. Alternatively, a urea or sulfonamide was incorporated in place of the acylated amine $(\mathbf{2 0}, \mathbf{2 1}, \mathbf{2 2})$ but resulted in loss of AAK1 affinity. The cyclopropanecarboxamide was found to be optimal (14). Minor changes, such as ring opening of the cyclopropane (16) or expansion to a cyclobutane (17) were found to result in modest loss of AAK1 affinity.

Table 1. SAR of 3-acylaminoindazole aryl ring.

\begin{tabular}{|c|c|c|}
\hline Cmpd & $\mathbf{R}$ & $\mathrm{AAK}_{1} \mathrm{IC}_{50}(\mathrm{nM})^{1}$ \\
\hline 1 & $3-\mathrm{NHSO}_{2} \mathrm{Me}$ & 220 \\
\hline 2 & 4- $\mathrm{NHSO}_{2} \mathrm{Me}$ & 1200 \\
\hline 3 & $3-\mathrm{CONH}_{2}$ & 3800 \\
\hline 4 & 4- $\mathrm{CONH}_{2}$ & 910 \\
\hline 5 & 3-NHAc & 1100 \\
\hline 6 & $3-\mathrm{CO}_{2} \mathrm{H}$ & 18000 \\
\hline 7 & $4-\mathrm{CO}_{2} \mathrm{H}$ & 2800 \\
\hline 8 & $3-\mathrm{OH}$ & 350 \\
\hline 9 & $4-\mathrm{OH}$ & 280 \\
\hline 10 & $3-\mathrm{NH}_{2}$ & 800 \\
\hline 11 & $3-\mathrm{N}\left(\mathrm{CH}_{3}\right) \mathrm{SO}_{2} \mathrm{Me}$ & 120 \\
\hline 12 & $3-\mathrm{CH}_{2} \mathrm{NHSO}_{2} \mathrm{Me}$ & 71 \\
\hline 13 & $3-\mathrm{NHSO}_{2}$ (isopropyl) & 54 \\
\hline 14 & 3-NHSO 2 (cyclopropyl) & 31 \\
\hline
\end{tabular}

${ }^{1}$ Each test compound was screened in AAK1 split luciferase assay in duplicate $(n=2)$ against AAK1 in dose-response (8-pt curve).

Based on the initial SAR, two additional 3-acylaminoindazoles (23 and 24) were prepared (Table 3) incorporating the aryl 3-cyclopropanecarboxamide that was found to yield potent inhibitors of AAK1. The analogs were designed to probe whether an alkyl amine or longer chain hydrocarbon could be tolerated at the indazole 3-position. Like lead compound 14, these analogs demonstrated promising inhibition of AAK 1 and BMP2K in the split luciferase assay $(93 \%$ I and $94 \%$ I of AAK 1 at $1 \mu \mathrm{M}$ for $\mathbf{2 3}$ and $\mathbf{2 4}$, respectively). TR-FRET binding-displacement assays were employed to further interrogate the biochemical selectivity of these promising 3-aminoacylindazoles across the four NAK family members (Table 3). ${ }^{21} \mathbf{2 3}$ and 24 were found to be dual AAK1/BMP2K inhibitors with more than 5-fold selectivity over STK16 and more than 50-fold selectivity over GAK.

To understand the selectivity of $\mathbf{2 3}$ and $\mathbf{2 4}$ across the NAK family kinases, co-crystal structures were solved with the BMP2K kinase domain (KD, Figure 2, Table S1). The BMP2K co-crystal structures were solved to $\sim 2.4 \AA$ resolution and 
showed that the core indazole formed the critical interaction with the hinge region of the kinase domain.

Table 2. SAR of 3-acylaminoindazole carboxamide.

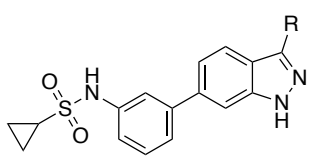

14

$\operatorname{AAK1~IC~}_{50}(\mathbf{n M})^{1}$

18000

${ }^{1}$ Each test compound was screened in duplicate $(n=2)$ in AAK1 split luciferase assay in dose-response (8-pt curve).

Given the high structural and sequence conservation between the ATP-binding sites of the BMP2K and AAK1 kinase domains (Figure 2), it is likely that both kinases bind to the indazole inhibitors using common interactions. The pendant aryl ring is accommodated by the hydrophobic region adjacent to the gatekeeper residue (residue Met130/126, BMP2K/AAK1 numbering) within the kinase ATP-binding site. These co-crystal structures suggest that large groups attached to the 4-position of the aryl ring (such as in $\mathbf{2}$ and $\mathbf{4}$ ) are likely not tolerated due to steric hinderance. Smaller groups at the 3- and 4-positions that can act as H-bond donors (such as in $\mathbf{8}$ and 9) can make favorable interactions with the DFG motif aspartate residue (Asp198/194, BMP2K/AAK1 numbering), whereas small acidic groups at these positions (such as in $\mathbf{6}$ and 7) are not favored due to charge repulsion. The structures also reveal that a sulfonamide at the 3-position can form $\mathrm{H}$-bonds with multiple polar residues within or adjacent to the protein ATP-binding pocket (Gln137/133, Asn185/181 and Asp198/194, BMP2K/AAK1 numbering). Groups attached to this sulfonamide are accommodated by a small cavity within the protein Ploop. When considering substituents attached to the indazole ring, poor binding is observed for compounds bearing sulfonamide groups in place of an amide (21 and 22). This finding is due to steric clash and electronic effects caused by the proximity of the sulfonamide oxygen atoms to nearby protein side chains. The co-crystal structures also reveal that groups attached to the 3-position amide are solvent exposed, an observation that helps rationalize the moderate tolerance of AAK1 for larger and bulkier substituents at this position (15, 17 and 19).

Guided by the co-crystal structures, analogs were designed with a focus on modifications to the sulfonamide portion of the molecule. Sulfonamides with pendant alkyl chains, alkyl amines, fluorinated alkyl chains, small hydrocarbon-based rings, and fluorinated aryl rings were synthesized (Table 4). Compounds built on Scaffold B were prepared using the chemistry in Scheme 1. Those built on Scaffold A were prepared using a slightly modified route. Briefly, reaction of the 3-aminophenylboronic acid pinacol ester with respective sulfonyl chlorides to furnish the sulfonamide-bearing boronic acid pinacol esters was found to be high yielding. This intermediate was coupled to the acylated indazole (I), prepared in Scheme 1, using Suzuki conditions. The analogs (25-39) were evaluated for potency and selectivity across the NAK family TR-FRET binding assays. Furthermore, cellular target engagement of AAK1, BMP2K, GAK and STK16 were determined by NanoBRET (NB) assays, where the respective kinases were fused to $19-\mathrm{kDa}$ luciferase (NLuc) and transiently expressed in HEK293 cells, and then incubated with a cell-permeable fluorescent energy transfer probe (tracer). ${ }^{20}$ Using increasing concentrations of test compounds dose-dependent displacement of tracer was observed, allowing calculation of the $\mathrm{IC}_{50}$ values in Table 4 . These NB assays enabled measurement of potency and selectivity of the compounds across the NAK family in living cells (Figure S1).

Table 3. NAK family TR-FRET data.

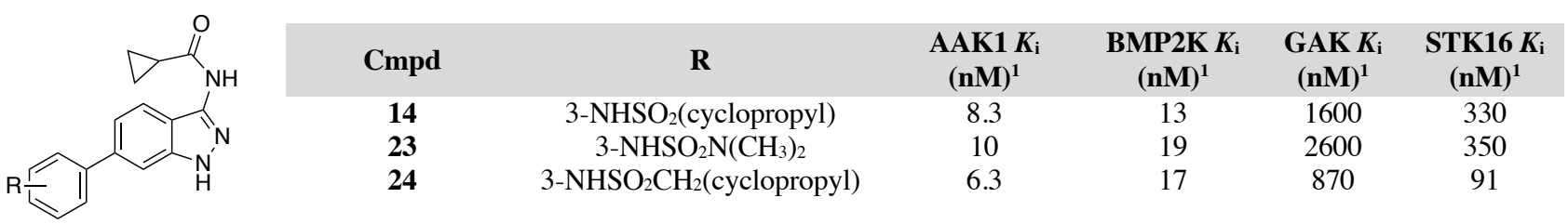

${ }^{1}$ Each test compound was screened in duplicate $(\mathrm{n}=2)$ in dose-response (16-pt curve). $\mathrm{IC}_{50}$ values were converted to $K_{\mathrm{i}}$ values using the Cheng Prusoff equation and the concentration and $K_{\mathrm{D}}$ values for the tracer. 


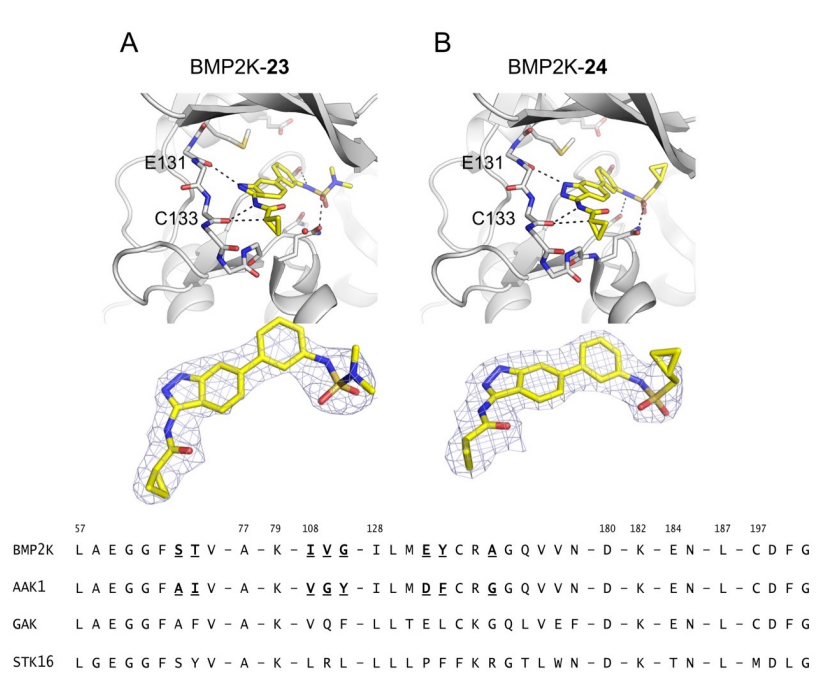

Figure 2. Co-crystal structures of 23 (A) and 24 (B) bound to the BMP2K-KD. Main-chain atoms for residues (131-137) within the $\mathrm{BMP} 2 \mathrm{~K}-\mathrm{KD}$ hinge region are represented as sticks. The side-chain atoms for BMP2K-KD gatekeeper reside (Met130) and those taking part in polar interactions with the ligands are shown as sticks. Black dashed lines depict possible hydrogen bonds between protein and ligand atoms. Red spheres denote the position of crystallographic water molecules. The bottom portion of each panel shows the chemical structure and $2 \mathrm{Fo}-\mathrm{Fc}$ electron density maps (contoured at $1.0 \sigma$ ) for each ligand. Active site sequence alignment for
NAK family included below with non-identical AAK1/BMP2K residues bolded and underlined. Residue numbers shown on the top row are for BMP2K.

Analysis of the data from both the biochemical and cellular assays revealed some notable trends and highlighted promising compounds as potential chemical probes. All of the analogs (25-39) were found to be dual AAK1/BMP2K inhibitors. Insertion of the methylene spacer between the aryl ring and sulfonamide (scaffold B) reduced potency for AAK1/BMP2K. Within the scaffold B group of molecules, incorporation of an alkyl chain capped with fluorines $(33,34,35)$ increased the in vitro biochemical affinity for GAK, but not sufficiently to demonstrate GAK activity in cells (Table S2). In previous work, we had also observed that only low nanomolar inhibitors of GAK possessed cellular activity. ${ }^{21}$ Almost all of the analogs were also devoid of activity in the STK16 NB assays. Several single-digit nanomolar biochemical inhibitors of AAK1 were prepared based upon scaffold A that also demonstrated target engagement with $\mathrm{IC}_{50}$ values between $190-1020 \mathrm{nM}$. Incorporation of fluorinated aryl rings was tolerated by AAK1 and BMP2K but not by GAK or STK16 $(\mathbf{2 9}, \mathbf{3 0}, \mathbf{3 1})$.

Scheme 2. Route to prepare compounds in Tables 3 and $4 .^{\mathrm{a}}$

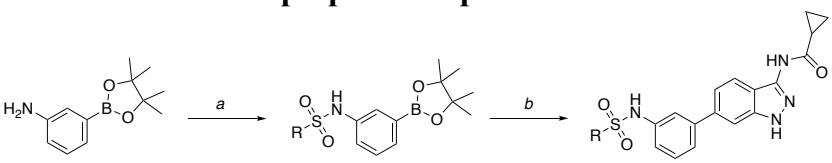

aReagents and conditions: (a) R-sulfonylchloride, pyridine, $0^{\circ} \mathrm{C}$ (49-65\%) (b) I, $\mathrm{Pd}(\mathrm{dppf}) \mathrm{Cl}_{2}, \mathrm{Na}_{2} \mathrm{CO}_{3}$, dioxane, $120^{\circ} \mathrm{C}$ (36-66\%).

Table 4. SAR of 3-acylaminoindazole probe candidates.
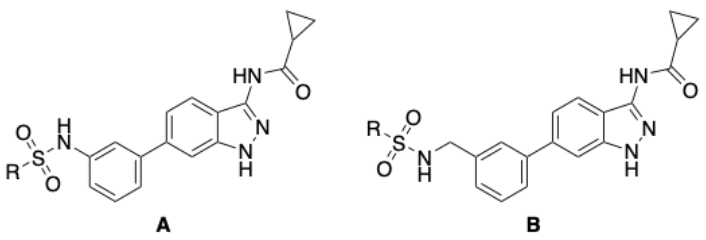

\begin{tabular}{|c|c|c|c|c|c|c|c|c|c|c|c|}
\hline \multirow{2}{*}{ Cmpd } & \multirow{2}{*}{ Scaffold } & \multirow{2}{*}{$\mathbf{R}$} & \multicolumn{2}{|c|}{ AAK1 (nM) } & \multicolumn{2}{|c|}{ BMP2K (nM) } & \multicolumn{2}{|c|}{ GAK (nM) } & \multicolumn{2}{|c|}{ STK16 (nM) } & \multirow{2}{*}{$\mathrm{S}_{10}(1 \mu \mathrm{M})$} \\
\hline & & & $K_{i}{ }^{1}$ & $\mathrm{IC}_{50}{ }^{2}$ & $K_{i}^{1}$ & $\mathrm{IC}_{50}{ }^{2}$ & $K_{i}^{1}$ & $\mathrm{IC}_{50}{ }^{3}$ & $K_{i}^{1}$ & $\mathrm{IC}_{50^{3}}{ }^{3}$ & \\
\hline 14 & A & cyclopropyl & 8.3 & 641 & 13 & 1420 & 1600 & $>10000$ & 330 & $>10000$ & 0.005 \\
\hline 25 & A & $\mathrm{N}\left(\mathrm{CH}_{2} \mathrm{CH}_{3}\right)_{2}$ & 9.1 & 770 & 17 & 2800 & 1700 & $>10000$ & 270 & $>10000$ & 0.02 \\
\hline 26 & A & $\mathrm{N}\left(\mathrm{CH}_{2} \mathrm{CH}_{3}\right)\left(\mathrm{CH}_{3}\right)$ & 8.2 & 375 & 20 & 1890 & 1700 & $>10000$ & 310 & $>10000$ & 0.017 \\
\hline 27 & A & $\mathrm{CH}_{2} \mathrm{CH}\left(\mathrm{CH}_{3}\right)_{2}$ & 6.2 & 490 & 17 & 1490 & 1300 & $>10000$ & 78 & $>10000$ & 0.002 \\
\hline 28 & A & $\mathrm{CH}_{2} \mathrm{CH}_{2} \mathrm{CH}_{3}$ & 12 & 655 & 30 & 2180 & 1100 & 9250 & 110 & $>10000$ & 0.01 \\
\hline 29 & A & 4-F-benzyl & 59 & 1020 & 120 & 2950 & 7000 & 9240 & 430 & $>10000$ & 0.007 \\
\hline 30 & A & 2-F-benzyl & 22 & 528 & 51 & 1620 & 4600 & $>10000$ & 470 & $>10000$ & 0.022 \\
\hline 31 & A & 3-F-benzyl & 24 & 379 & 53 & 1190 & 4600 & $>10000$ & 510 & $>10000$ & 0.017 \\
\hline 32 & A & cyclobutyl & 8.5 & 192 & 17 & 707 & 2900 & $>10000$ & 240 & $>10000$ & 0.007 \\
\hline 33 & $\mathrm{~B}$ & $\mathrm{CH}_{2} \mathrm{CH}_{2} \mathrm{CF}_{3}$ & 32 & 2710 & 27 & 1890 & 510 & $>10000$ & 180 & $>10000$ & 0.022 \\
\hline 34 & B & $\mathrm{N}\left(\mathrm{CH}_{2} \mathrm{CF}_{3}\right)\left(\mathrm{CH}_{3}\right)$ & 44 & 3400 & 42 & 3030 & 780 & $>10000$ & 290 & $>10000$ & NT \\
\hline 35 & $\mathrm{~B}$ & $\mathrm{CH}_{2} \mathrm{CF}_{3}$ & 39 & 2350 & 33 & 1620 & 580 & $>10000$ & 180 & $>10000$ & 0.005 \\
\hline 36 & B & $\mathrm{CH}_{2} \mathrm{CH}\left(\mathrm{CH}_{3}\right)_{2}$ & 30 & 1530 & 59 & 6550 & 3900 & $>10000$ & 450 & $>10000$ & 0.002 \\
\hline 37 & $\mathrm{~B}$ & $\mathrm{~N}\left(\mathrm{CH}_{2} \mathrm{CH}_{3}\right)_{2}$ & 33 & 1470 & 74 & 3830 & 3200 & $>10000$ & 220 & $>10000$ & NT \\
\hline 38 & $\mathrm{~B}$ & $\mathrm{CH}_{2}$ (cyclopropyl) & 22 & 1260 & 37 & 2740 & 3200 & $>10000$ & 150 & $>10000$ & 0.01 \\
\hline 39 & B & $\mathrm{N}\left(\mathrm{CH}_{2} \mathrm{CH}_{3}\right)\left(\mathrm{CH}_{3}\right)$ & 20 & 1790 & 36 & 2960 & 360 & $>10000$ & 86 & $>10000$ & NT \\
\hline
\end{tabular}

${ }^{1}$ Each analog was screened in the TR-FRET in duplicate $(\mathrm{n}=2)$ and dose-response (16-pt curve). $\mathrm{IC}_{50}$ values were converted to $K_{\mathrm{i}}$ values using the Cheng Prusoff equation and the concentration and $K_{\mathrm{D}}$ values for the tracer. ${ }^{2} \mathrm{AAK} 1$ and BMP2K NB assays were performed in triplicate $(n=3)$ to provide $\mathrm{IC}_{50}$ values (11-pt curve). ${ }^{3} \mathrm{STK} 16$ and GAK NB assays performed in singlicate ( $\left.\mathrm{n}=1\right)$ based on demonstration of poor activity. ${ }^{4} \mathrm{~S}_{10}(1 \mu \mathrm{M})$ is a measure of selectivity equal to the percentage of the screenable human kinome biochemically inhibited by $>90 \%$ at $1 \mu \mathrm{M}$. 
The 3-fluoroaryl analog (31) was one of the most potent compounds in the cellular NB assays for AAK1 and BMP2K, while the 4-fluoro analog (29) was much less active on both kinases. Compounds bearing small hydrocarbon chains and rings or alkyl amines on the sulfonamide proved to be the best dual AAK1/BMP2K inhibitors biochemically $(\mathbf{1 4}, \mathbf{2 5}, \mathbf{2 6}, \mathbf{2 7}$, 28, 32) and demonstrated at least 35 -fold selectivity for BMP2K over GAK. The biochemical selectivity for BMP2K over STK16 was more variable, ranging from 4-16-fold. When considering the cell-based activity, 14, 26, 27, and 30-32 were the best performers in both the AAK1 and BMP2K NB, while $\mathbf{2 5}$ and $\mathbf{2 8}$ exhibited a slight drop in potency when transitioning from the biochemical to cell-based assays.

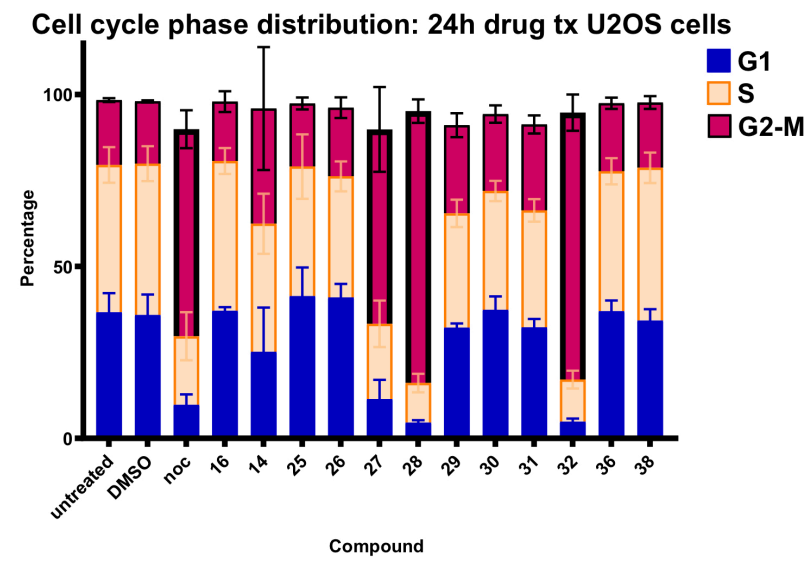

Figure 3. Cell cycle distributions. Cell cycle phases were determined by analytical flow cytometry of DNA content and DNA synthesis in U2OS cells after $24 \mathrm{~h}$ treatment with $5 \mu \mathrm{M}$ of the indicated compounds (error bars represent $n=3$ ). Note: compounds 27, 28, and $\mathbf{3 2}$ induce $\mathrm{G} 2 / \mathrm{M}$ arrest (nocodazole serves as a positive control for arrest with G2/M DNA content).

All of the compounds from Table 4 built upon Scaffold A and a few representative compounds from Scaffold B (25-33, 35, 36 and 38) were evaluated for selectivity by KINOMEscan (DiscoverX) against 403 wildtype human protein kinases at 1 $\mu \mathrm{M}$ (Table 4). ${ }^{22}$ The compounds demonstrated remarkable selectivity for AAK1/BMP2K across the 403 human kinases. 27 and 36 were found to selectivity inhibit only AAK1/BMP2K $\left(\mathrm{S}_{10}(1 \mu \mathrm{M})=0.002\right)$. The remaining analogs were all narrow spectrum inhibitors of AAK $1 / \mathrm{BMP} 2 \mathrm{~K}$ with $>90 \%$ inhibition of only $1-8$ additional kinases $\left(\mathrm{S}_{10}(1 \mu \mathrm{M})=0.005-0.01\right.$, Figure S4).

To explore the phenotypic effect of AAK1/BMP2K inhibition, the lead compound $\mathbf{1 4}$ and the inactive analog 16 were tested in several cell lines. Surprisingly, we observed that $\mathbf{1 4}$ had cytostatic effects on cells after $24 \mathrm{~h}$ of continuous treatment. Further study in U2OS human osteosarcoma cells showed that 14 elicited cell cycle arrest in $\mathrm{G} 2 / \mathrm{M}$ phase at 5-10 $\mu \mathrm{M}$ (Figure $\mathrm{S} 2$ ). The lack of effect of inactive analog 16 implied that the $\mathrm{G} 2 / \mathrm{M}$ arrest was not due to inhibition of AAK1/BMP2K but was likely due to inhibition of an off-target kinase. Cell growth inhibition and cell cycle arrest were observed in HEK293T cells as well (data not shown). Although the molecular mechanism of the cell cycle arrest proved to be elusive, we employed the U2OS cells as a screen to remove analogs that displayed this phenotype for consideration as chemical probes. A total of 12 analogs were screened for their effect on G2/M arrest (Figure
3). Nocondazole treatment was used as a positive control for a strong arrest in mitosis.

Only analogs 27, 28, and 32 demonstrated the collateral G2 or $\mathrm{M}$ phase arrest at $5 \mu \mathrm{M}$, while the remaining analogs were devoid of this phenotype (Figure 3, Figure S3). Compound 25 was selected as a chemical probe due to its potency on AAK1 and BMP2K, its kinome-wide selectivity, and its lack of a $\mathrm{G} 2 / \mathrm{M}$ arresting phenotype.

Following KINOMEscan of compound 25 (SGC-AAK-1), the binding affinity $\left(\mathrm{K}_{\mathrm{D}}\right)$ of all kinases inhibited $>80 \%$ at $1 \mu \mathrm{M}$ plus AAK1 and a few kinases inhibited by structurally related analogs was determined (Table S3). Only 3 kinases were bound by 25 within 30 -fold of the potency of $\mathbf{2 5}$ for AAK1: RIOK1 $\left(K_{\mathrm{D}}=72\right)$, RIOK3 $\left(K_{\mathrm{D}}=290\right)$ and PIP5K1C $\left(K_{\mathrm{D}}=260\right) .{ }^{23} \mathrm{In}$ accordance with our previously described probe criteria, ${ }^{24}$ SGC-AAK1-1 (25) is the best available dual AAK1/BMP2K chemical probe. Compound $\mathbf{1 6}$ (SGC-AAK1-1N) is the complimentary negative control analog.

We have described the design, synthesis and biological evaluation of a series of acylaminoindazoles as selective dual inhibitors of AAK1/BMP2K. Compound 25 emerged as the most selective and cell active compound within the series. Xray co-crystal structures of selected acylaminoindazoles bound to BMP2K revealed key interactions within the ATP binding pocket that explain the high affinity of this series for AAK1 and BMP2K. The effect of $\mathbf{2 5}$ on WNT signaling was recently reported. ${ }^{23}$ Further investigations into the effects of selective pharmacological inhibition of AAK1 are ongoing.

\section{ASSOCIATED CONTENT}

\section{Supporting Information}

Supplemental material is available free of charge via the Internet at http://pubs.acs.org.

Protein crystallization conditions, NanoBRET and cell cycle assay results, experimental methods, synthesis and characterization of target compounds $\mathbf{2 5}, \mathbf{2 6}, \mathbf{2 7}$, and $\mathbf{2 8}$ are included (PDF).

\section{Accession Codes}

The PDB accession codes for the X-ray co-crystal structures of $\mathrm{BMP} 2 \mathrm{~K}+\mathbf{2 3}$ and $\mathrm{BMP} 2 \mathrm{~K}+\mathbf{2 4}$ are $5 \mathrm{I} 3 \mathrm{O}$ and 5I3R, respectively.

\section{AUTHOR INFORMATION}

\section{Corresponding Author}

* Tel: 919-962-5349. E-mail: alison.axtman@unc.edu.

\section{ORCID}

Carrow I. Wells: 0000-0003-4799-6792

Rafael M. Couñago: 0000-0002-2826-1689

Juanita C. Limas: 0000-0001-6384-8787

Tuanny L. Almeida: 0000-0002-4528-9130

Jeanette G. Cook: 0000-0003-0849-7405

David H. Drewry: 0000-0001-5973-5798

Jonathan M. Elkins: 0000-0002-2826-1689

Opher Gileadi: 0000-0002-2826-1689

Nirav Kapadia: 0000-0002-2826-1689

Álvaro Lorente-Macías: 0000-0001-9510-714X

Julie E. Pickett: 0000-0002-9535-8528 
Roberta R. Ruela-de-Sousa: 0000-0002-3762-2247

Timothy M. Willson: 0000-0003-4181-8223

William J. Zuercher: 0000-0002-9836-0068

Alison D. Axtman: 0000-0003-4779-9932

\section{Notes}

The authors declare no competing financial interest.

\section{Author Contributions}

The manuscript was written through contributions of all authors. All authors have given approval to the final version of the manuscript.

\section{Funding Sources}

The Structural Genomics Consortium is a registered charity (number 1097737) that receives funds from AbbVie, Bayer Pharma AG, Boehringer Ingelheim, Canada Foundation for Innovation, Eshelman Institute for Innovation, Genome Canada, Innovative Medicines Initiative (EU/EFPIA) [ULTRA-DD grant no. 115766], Janssen, Merck KGaA Darmstadt Germany, MSD, Novartis Pharma AG, Ontario Ministry of Economic Development and Innovation, Pfizer, São Paulo Research Foundation-FAPESP [2013/50724-5, 2014/5087-0 and 2016/17469-0], Takeda, and Wellcome [106169/ZZ14/Z]. This work was also supported by the Brazilian agency CNPq (Conselho Nacional de Desenvolvimento Científico e Tecnológico) [465651/2014-3]. J.G.C is supported by grants from the NIH/ NIGMS (GM083024, and R25GM089569); JCL is supported by an HHMI Gilliam Fellowship for Advanced Study (GT10886) \& T32 award (T32GM007040). The UNC Flow Cytometry Core Facility is supported in part by P30 CA016086 Cancer Center Core Support Grant to the UNC Lineberger Comprehensive Cancer Center. Research reported in this publication was supported in part by the NC Biotech Center Institutional Support Grants 2017-IDG-1025 and 2018-IDG-1030, and by the NIH 1UM2AI30836-01. A.L.M. acknowledges support from the Spanish Ministry of Education, Culture and Sports (FPU grant ref. 14/00818). D.H.D. and R.N. acknowledge 1R44TR001916 for support.

\section{ACKNOWLEDGEMENT}

R.M.C. thanks Diamond Light Source for access to beamline I03 (proposal number MX14664) that contributed to the results presented here. Constructs for NanoBRET measurements of AAK1, BMP2K, GAK and STK16 were kindly provided by Promega.

\section{ABBREVIATIONS}

AAK1, AP2-associated protein kinase 1; BMP2K/BIKE, BMP2inducible kinase; STK16, serine/Threonine kinase 16; GAK, cyclin-G-associated kinase; NAK, numb-associated kinase; AP2, adaptor protein 2; TR-FRET, time-resolved fluorescence resonance energy transfer; PKIS, published kinase inhibitor set; DSF, differential scanning fluorimetry; ATP, adenosine 5'-triphosphate; SAR, structure-activity relationship; HEK, human embryonic kidney; NB, NanoBRET; U2OS, U2 osteosarcoma; KD, kinase domain; NLuc, Nanoluciferase; tx, treatment; H-bond, hydrogen-bond.

\section{REFERENCE}

1. Smythe, E.; Ayscough, K. R., The Ark1/Prk1 family of protein kinases. Regulators of endocytosis and the actin skeleton. EMBO reports $\mathbf{2 0 0 3 , ~} 4$ (3), 246-51.

2. Sorrell, F. J.; Szklarz, M.; Abdul Azeez, K. R.; Elkins, J. M.; Knapp, S., Family-wide Structural Analysis of Human Numb-Associated Protein Kinases. Structure 2016, 24 (3), 401-11. 3. Conner, S. D.; Schmid, S. L., Identification of an adaptor-associated kinase, AAK1, as a regulator of clathrin- mediated endocytosis. The Journal of cell biology 2002, 156 (5), 921-9.

4. Henderson, D. M.; Conner, S. D., A novel AAK1 splice variant functions at multiple steps of the endocytic pathway. Molecular biology of the cell 2007, 18 (7), 2698-706.

5. Neveu, G.; Barouch-Bentov, R.; Ziv-Av, A.; Gerber, D.; Jacob, Y.; Einav, S., Identification and targeting of an interaction between a tyrosine motif within hepatitis $\mathrm{C}$ virus core protein and AP2M1 essential for viral assembly. PLoS Pathog 2012, 8 (8), e1002845.

6. Gupta-Rossi, N.; Ortica, S.; Meas-Yedid, V.; Heuss, S.; Moretti, J.; Olivo-Marin, J. C.; Israel, A., The adaptor-associated kinase 1 , AAK1, is a positive regulator of the Notch pathway. $J$ Biol Chem 2011, 286 (21), 18720-30.

7. Sorensen, E. B.; Conner, S. D., AAK1 regulates Numb function at an early step in clathrin-mediated endocytosis. Traffic (Copenhagen, Denmark) 2008, 9 (10), 1791-800.

8. $\quad$ Borner, G. H.; Antrobus, R.; Hirst, J.; Bhumbra, G. S.; Kozik, P.; Jackson, L. P.; Sahlender, D. A.; Robinson, M. S., Multivariate proteomic profiling identifies novel accessory proteins of coated vesicles. The Journal of cell biology 2012, 197 (1), 141-60.

9. $\quad$ Krieger, J. R.; Taylor, P.; Gajadhar, A. S.; Guha, A.; Moran, M. F.; McGlade, C. J., Identification and selected reaction monitoring (SRM) quantification of endocytosis factors associated with Numb. Molecular \& cellular proteomics : MCP 2013, 12 (2), 499-514.

10. Shi, B.; Conner, S. D.; Liu, J., Dysfunction of endocytic kinase AAK1 in ALS. International journal of molecular sciences 2014, 15 (12), 22918-32.

11. Abdel-Magid, A. F., Inhibitors of Adaptor-Associated Kinase 1 (AAK1) May Treat Neuropathic Pain, Schizophrenia, Parkinson's Disease, and Other Disorders. ACS medicinal chemistry letters 2017, 8 (6), 595-597.

12. Liu, H. P.; Lin, Y.J.; Lin, W. Y.; Wan, L.; Sheu, J. J.; Lin, H. J.; Tsai, Y.; Tsai, C. H.; Tsai, F. J., A novel genetic variant of BMP2K contributes to high myopia. J Clin Lab Anal 2009, 23 (6), 362-7.

13. Zhou, H.; Xu, M.; Huang, Q.; Gates, A. T.; Zhang, X. D.; Castle, J. C.; Stec, E.; Ferrer, M.; Strulovici, B.; Hazuda, D. J.; Espeseth, A. S., Genome-scale RNAi screen for host factors required for HIV replication. Cell host \& microbe 2008, 4 (5), 495504.

14. Kostich, W.; Hamman, B. D.; Li, Y.-W.; Naidu, S.; Dandapani, K.; Feng, J.; Easton, A.; Bourin, C.; Baker, K.; Allen, J.; Savelieva, K.; Louis, J. V.; Dokania, M.; Elavazhagan, S.; Vattikundala, P.; Sharma, V.; Lal Das, M.; Shankar, G.; Kumar, A.; Holenarsipur, V. K.; Gulianello, M.; Molski, T.; Brown, J. M.; Lewis, M. C.; Huang, Y.; Lu, Y.; Pieschl, R.; O'Malley, K.; Lippy, J. S.; Nouraldeen, A.; Lanthorn, T. H.; Ye, G.; WIlson, A.; Balakrishnan, A.; Denton, R.; Grace, J. E.; Lentz, K. A.; Santone, K. S.; Bi, Y.; Main, A.; Swaffield, J.; Carson, K.; Mandlekar, S.; Vikramadithyan, R. K.; Nara, S. J.; Dzierba, C.; Bronson, J.; Macor, J. E.; Zaczek, R.; Westphal, R.; Kiss, L.; Bristow, L.; Conway, C. M.; Zambrowicz, B.; Albright, C. F., Inhibition of AAK1 kinase as a novel therapeutic approach to treat neuropathic pain. J Pharmacol Exp Ther 2016, 358 (3), 371-386.

15. Chaikuad, A.; Keates, T.; Vincke, C.; Kaufholz, M.; Zenn, M.; Zimmermann, B.; Gutierrez, C.; Zhang, R. G.; HatzosSkintges, C.; Joachimiak, A.; Muyldermans, S.; Herberg, F. W.; Knapp, S.; Muller, S., Structure of cyclin G-associated kinase (GAK) trapped in different conformations using nanobodies. Biochem J 2014, 459 (1), 59-69.

16. Eswaran, J.; Bernad, A.; Ligos, J. M.; Guinea, B.; Debreczeni, J. E.; Sobott, F.; Parker, S. A.; Najmanovich, R.; Turk, B. E.; Knapp, S., Structure of the human protein kinase 
MPSK1 reveals an atypical activation loop architecture. Structure 2008, 16 (1), 115-24.

17. Elkins, J. M.; Fedele, V.; Szklarz, M.; Abdul Azeez, K. R.; Salah,E.; Mikolajczyk, J.; Romanov, S.; Sepetov, N.; Huang, X. P.; Roth, B. L.; Al Haj Zen, A.; Fourches, D.; Muratov, E.; Tropsha, A.; Morris, J.; Teicher, B. A.; Kunkel, M.; Polley, E.; Lackey, K. E.; Atkinson, F. L.; Overington, J. P.; Bamborough, P.; Muller, S.; Price, D. J.; Willson, T. M.; Drewry, D. H.; Knapp, S.; Zuercher, W. J., Comprehensive characterization of the Published Kinase Inhibitor Set. Nature biotechnology 2016, 34 (1), 95-103.

18. Jester, B. W.; Cox, K. J.; Gaj, A.; Shomin, C. D.; Porter, J. R.; Ghosh, I., A coiled-coil enabled split-luciferase threehybrid system: applied toward profiling inhibitors of protein kinases. J Am Chem Soc 2010, 132 (33), 11727-35.

19. Jester, B. W.; Gaj, A.; Shomin, C. D.; Cox, K. J.; Ghosh, I., Testing the promiscuity of commercial kinase inhibitors against the AGC kinase group using a split-luciferase screen. Journal of medicinal chemistry 2012, 55 (4), 1526-37.

20. Vasta, J. D.; Corona, C. R.; Wilkinson, J.; Zimprich, C. A.; Hartnett, J. R.; Ingold, M. R.; Zimmerman, K.; Machleidt, T.; Kirkland, T. A.; Huwiler, K. G.; Ohana, R. F.; Slater, M.; Otto, P.; Cong, M.; Wells, C. I.; Berger, B. T.; Hanke, T.; Glas, C.; Ding, K.; Drewry, D. H.; Huber, K. V. M.; Willson, T. M.; Knapp, S.; Muller, S.; Meisenheimer, P. L.; Fan, F.; Wood, K. V.; Robers, M. B., Quantitative, Wide-Spectrum Kinase Profiling in Live Cells for Assessing the Effect of Cellular ATP on Target Engagement. Cell chemical biology 2017, 25 (2), 206-214.
21. Asquith, C. R. M.; Laitinen, T.; Bennett, J. M.; Godoi, P. H.; East, M. P.; Tizzard, G. J.; Graves, L. M.; Johnson, G. L.; Dornsife, R. E.; Wells, C. I.; Elkins, J. M.; Willson, T. M.; Zuercher, W. J., Identification and Optimization of 4Anilinoquinolines as Inhibitors of Cyclin G Associated Kinase. ChemMedChem 2018, 13 (1), 48-66.

22. Davis, M. I.; Hunt, J. P.; Herrgard, S.; Ciceri, P.; Wodicka, L. M.; Pallares, G.; Hocker, M.; Treiber, D. K.; Zarrinkar, P. P., Comprehensive analysis of kinase inhibitor selectivity. Nature biotechnology 2011, 29 (11), 1046-51.

23. Agajanian, M. J.; Walker, M.P.; Axtman, A. D.; Ruelade-Sousa, R. R.; Serafin, D. S.; Rabinowitz, A. D.; Graham, D. M.; Ryan, M. B.; Tamir, T.; Nakamichi, Y.; Gammons, M. V.; Bennett, J. M.; Counago, R. M.; Drewry, D. H.; Elkins, J. M.; Gileadi, C.; Gildadi, O.; Godoi, P. H.; Kapadia, N.; Muller, S.; Santiago, A. S.; Sorrell, F. J.; Wells, C. I.; Fedorov, O.; Willson, T. M.; Zuercher, W. J.; Major, M. B., WNT activates the AAK1 kinase to promote clathrin-mediated endocytosis of LRP6 and establish a negative feedback loop. Cell reports 2019, 26 (1), 7983.

24. Asquith, C. R. M.; Berger, B.-T.; Wan, J.; Bennett, J. M.; Capuzzi, S. J.; Crona, D. J.; Drewry, D. H.; East, M. P.; Elkins, J. M.; Fedorov, O.; Godoi, P. H.; Hunter, D. M.; Knapp, S.; Müller, S.; Torrice, C. D.; Wells, C. I.; Earp, H. S.; Willson, T. M.; Zuercher, W. J., SGC-GAK-1: A Chemical Probe for Cyclin G Associated Kinase (GAK). Journal of medicinal chemistry 2019, 62 (5), 2830-2836. 